\title{
Identification and Characterization of Carbendazim-Resistant Isolates of Gibberella zeae
}

\author{
Xin Liu, Yanni Yin, Jianbing Wu, Jinhua Jiang, and Zhonghua Ma, Key Laboratory of Molecular Biology of \\ Crop Pathogens and Insects, Institute of Biotechnology, Zhejiang University, Hangzhou 310029, China
}

\begin{abstract}
Liu, X., Yin, Y., Wu, J., Jiang, J., and Ma, Z. 2010. Identification and characterization of carbendazim-resistant isolates of Gibberella zeae. Plant Dis. 94:1137-1142.

Sensitivity of Gibberella zeae to carbendazim was determined by measuring mycelial growth in fungicide-amended media. Among 1,529 isolates tested, 31 isolates showed a high level of resistance (HR) to carbendazim (fungicide concentration that results in $50 \%$ inhibition of mycelial growth [ $\left.\mathrm{EC}_{50}\right]$ of 10.35 to $30.26 \mathrm{mg}$ a.i. liter ${ }^{-1}$ ) and 10 isolates were moderately resistant (MR) $\left(\mathrm{EC}_{50}\right.$ of 4.50 to $7.28 \mathrm{mg}$ a.i. liter ${ }^{-1}$ ). The remaining 1,488 isolates were sensitive to carbendazim and were unable to grow on potato dextrose agar amended with carbendazim at $2 \mathrm{mg}$ a.i. liter $^{-1}$. Analysis of DNA sequences of the $\beta 2$-tubulin (Tub2) gene showed that all 10 MR isolates had a point mutation at codon 198 causing a replacement of glutamic acid by glutamine. At the codon position 167, the amino acid phenylalanine was replaced by tyrosine in 28 of $31 \mathrm{HR}$ isolates. The remaining three HR isolates had a point mutation at codon 200 which converted phenylalanine to tyrosine. Based on these point mutations in the Tub2 gene, allele-specific polymerase chain reaction primers were developed for rapid detection of the point mutations. The rapid molecular method will be a valuable tool for the monitoring of carbendazim resistance in $G$. zeae. Additionally, deletion of the $\beta 1$-tubulin gene (Tub1) in the HR isolate GJ33 resulted in increased resistance to carbendazim. These results indicate that $T u b l$ plays a role in the sensitivity of $G$. zeae to carbendazim.
\end{abstract}

Fusarium head blight (FHB), caused by Gibberella zeae, is a devastating disease of wheat in China. Disease epidemics can lead to significant yield loss in the more than 5 million hectares of wheat grown each year. From 1991 to 2007, there were two severe and seven moderate epidemics of FHB in the middle to lower reaches of the Yangtze River. In 2003, the proportion of scabbed spikes was 60 to $80 \%$ in this region, which resulted in greater than $20 \%$ yield losses (18). Because most wheat cultivars planted in China are susceptible to $G$. zeae, methyl benzimidazole carbamate (MBC) fungicides, particularly carbendazim and thiophanate-methyl, have been used extensively to control FHB during the past three decades. Because MBC-resistant isolates of G. zeae were first detected in 1992 in China, a decline in the control of FHB by MBC has been observed frequently in the Zhejiang and Jiangsu Provinces (3). Therefore, in order to control FHB effectively, it is necessary to monitor the development of $\mathrm{MBC}$-resistant $G$. zeae populations in China.

Corresponding author: Z. Ma

E-mail: zhma@zju.edu.cn

Accepted for publication 26 May 2010.

doi:10.1094/PDIS-94-9-1137

(C) 2010 The American Phytopathological Society
Resistance to MBC fungicides has been reported for many phytopathogenic fungi, including Botrytis cinerea, Cladobotryum dendroides, Helminthosporium solani, Monilinia fructicola, M. laxa, Tapesia yallundae, and Venturia inaequalis $(1,7,8,12)$. In most cases, the resistance is caused by point mutations in the $\beta$-tubulin gene which result in altered amino acid sequences at the MBC-binding site (12). In G. zeae, two homologous $\beta$-tubulin genes (Tub1 and Tub2) exist in the fungal genome. A previous study showed that resistant and sensitive isolates of G. zeae had identical sequences of Tubl (3). The resistance of G. zeae to $\mathrm{MBC}$ is primarily due to a single point mutation at codons 167 or 200 in Tub2 (FGSG 06611) (4,11). Double mutations at codons 73 and 198 in Tub2 were also detected from a highly resistant isolate but the frequency of this genotype was very low in field populations (4). Many previous studies have shown that changes at codons 6, 50, 167, 198, 200, and 240 in the $\beta$-tubulin gene could cause MBC resistance in several pathogenic fungi (12). Thus, it is necessary to determine whether such mutations also occur in G. zeae.

The objectives of this study were to (i) screen carbendazim-resistant isolates from a collection of 1,529 isolates of G. zeae, (ii) investigate molecular mechanisms of carbendazim resistance in field isolates of G. zeae, (iii) develop a molecular technique for the rapid detection of alleles mediating $\mathrm{MBC}$ resistance in G. zeae, and (iv) investigate the contribution of Tubl to the sensitivity of G. zeae to carbendazim.

\section{MATERIALS AND METHODS}

Survey of sensitivity of $G$. zeae to carbendazim. In total, 1,529 single-spore isolates of G. zeae used in this study were collected from six provinces of China, including Anhui, Jiangsu, Hebei, Henan, Shandong, and Zhejiang, in 2008 and 2009 (Table 1). Each isolate was recovered from a single infected wheat head and no more than 10 isolates were collected from a single field.

Carbendazim $(50 \%$ a.i.; Sicuan GuoGuang Agricultural Chemical Company Ltd., China) was suspended in sterilized water and adjusted to $100 \mathrm{mg}$ a.i. liter $^{-1}$. The fungicide was added to potato dextrose agar (PDA) after autoclaving at the following concentrations: $0,2,10,50,100$, or $500 \mathrm{mg}$ a.i. liter ${ }^{-1}$. Single $5-\mathrm{mm}$ mycelial plugs were taken from the edge of a 2day-old colony of each isolate and placed on each concentration of fungicideamended PDA. Four replicated plates were used for each fungicide concentration. Petri plates were incubated at $25^{\circ} \mathrm{C}$ for 3 days in the dark and then radial growth (in millimeters) of each isolate was measured, with the original mycelial plug diameter (5 $\mathrm{mm}$ ) subtracted from this measurement. The experiment was repeated.

The fungicide concentration that results in $50 \%$ inhibition of mycelial growth $\left(\mathrm{EC}_{50}\right)$ for 10 sensitive and 41 resistant isolates identified above was determined by assessing radial growth on PDA plates amended with carbendazim at $0,0.25,0.5$, $1,2,4,8,16,32,64$, and $128 \mathrm{mg}$ a.i liter $^{-1}$. There were three replicates for each isolate at each concentration. After the plates were incubated at $25^{\circ} \mathrm{C}$ for 3 days in the dark, radial growth (in millimeters) of each isolate was measured as described above. The experiment was performed three times. For each plate, the average colony diameter of each isolate at each fungicide concentration was used for calculation of the $\mathrm{EC}_{50}$ value with a data processing system (Hangzhou Reifeng Information Technology Ltd., Hangzhou, China).

Analysis of DNA sequences of Tub2 from carbendazim-resistant isolates of G. zeae. Six highly resistant (HR) isolates (HA29, HA36, HA70, HN40, HY11, and GJ33), two moderately resistant (MR) isolates (HA34 and XS19), and one sensitive isolate (PH-1) were used for DNA 
sequence analysis of Tub2. Fungal DNA was extracted by using a previously published protocol (16). Briefly, approximately $100 \mathrm{mg}$ of mycelia of each isolate were harvested from PDA plates, transferred to a $1.5-\mathrm{ml}$ microtube, and ground with a motor-driven glass pestle in $1 \mathrm{ml}$ of lysis buffer (200 mM Tris- $\mathrm{HCl}, 50 \mathrm{mM}$ ethylenediaminetetraacetic acid, $200 \mathrm{mM}$ $\mathrm{NaCl}$, and $1 \%$ (wt/vol) n-lauroylsarcosine sodium salt, $\mathrm{pH}$ 8.0). The mixture was incubated for $10 \mathrm{~min}$ at room temperature before being centrifuged at $18,000 \times g$ for $5 \mathrm{~min}$ at $4^{\circ} \mathrm{C}$. The supernatant $(800 \mathrm{ml})$ was transferred to a clean microtube and mixed with an equal volume of isopropanol. The resulting DNA pellet was precipitated by centrifugation at $18,000 \times g$ for 2 min at $4^{\circ} \mathrm{C}$. After a wash with $70 \%$ ethanol, the DNA pellet was air dried and dissolved in $100 \mathrm{ml}$ of $100 \mathrm{mM}$ Tris- $\mathrm{HCl}$ buffer ( $\mathrm{pH}$ 8.0).

Table 1. Survey of the sensitivity to carbendazim in Gibberella zeae isolates collected from different locations in China

\begin{tabular}{|c|c|c|c|}
\hline Origin (city, province) & $\begin{array}{l}\text { No. of } \\
\text { isolates }\end{array}$ & $\begin{array}{c}\text { Year of } \\
\text { isolation }\end{array}$ & $\begin{array}{l}\text { No. of carbendazim-resistant } \\
\text { isolates }\end{array}$ \\
\hline Anqing, Anhui & 24 & 2009 & 0 \\
\hline Bengbu, Anhui & 1 & 2008 & 0 \\
\hline Bengbu, Anhui & 66 & 2009 & 0 \\
\hline Chaohu, Anhui & 90 & 2009 & 0 \\
\hline Chuzhou, Anhui & 81 & 2009 & 0 \\
\hline Fuyang, Anhui & 1 & 2008 & 0 \\
\hline Fuyang, Anhui & 160 & 2009 & 1 \\
\hline Hefei, Anhui & 3 & 2008 & 0 \\
\hline Huainan, Anhui & 2 & 2008 & 0 \\
\hline Huainan, Anhui & 26 & 2009 & 0 \\
\hline Liu’an, Anhui & 20 & 2008 & 0 \\
\hline Liu'an, Anhui & 21 & 2009 & 0 \\
\hline Ningguo, Anhui & 3 & 2008 & 0 \\
\hline Ningguo, Anhui & 19 & 2009 & 0 \\
\hline Tongcheng, Anhui & 15 & 2009 & 0 \\
\hline Wuhu, Anhui & 3 & 2008 & 0 \\
\hline Xuanzhou, Anhui & 3 & 2008 & 0 \\
\hline Hai'an, Jiangsu & 80 & 2008 & 4 \\
\hline Hai'an, Jiangsu & 170 & 2009 & 12 \\
\hline Jingjiang, Jiangsu & 42 & 2008 & 2 \\
\hline Ruancheng, Hebei & 30 & 2009 & 0 \\
\hline Shenzhou, Hebei & 50 & 2009 & 0 \\
\hline Xinji, Hebei & 28 & 2009 & 0 \\
\hline Jiaozuo, Henan & 22 & 2009 & 0 \\
\hline Luohe, Henan & 52 & 2009 & 0 \\
\hline Luoyang, Henan & 2 & 2009 & 0 \\
\hline Tai'an, Shandong & 102 & 2009 & 0 \\
\hline Haining, Zhejiang & 74 & 2009 & 2 \\
\hline Haining, Zhejiang & 10 & 2008 & 4 \\
\hline Haiyan, Zhejiang & 9 & 2008 & 0 \\
\hline Haiyan, Zhejiang & 238 & 2009 & 12 \\
\hline Hangzhou, Zhejiang & 20 & 2009 & 0 \\
\hline Jiaxing, Zhejiang & 12 & 2008 & 3 \\
\hline Xiaoshan, Zhejiang & 50 & 2008 & 1 \\
\hline
\end{tabular}

The primer pair Tub2-seq-F + Tub2-seq$\mathrm{R}$ (Table 2) was designed to amplify a partial sequence of Tub2. Polymerase chain reaction (PCR) was performed with the following parameters: preheating for 3 min at $95^{\circ} \mathrm{C}$ and 35 cycles of denaturation at $94^{\circ} \mathrm{C}$ for $40 \mathrm{~s}, 55^{\circ} \mathrm{C}$ for $40 \mathrm{~s}, 72^{\circ} \mathrm{C}$ for $1.5 \mathrm{~min}$, and $72^{\circ} \mathrm{C}$ for $5 \mathrm{~min}$. Using primer pair Tub2-seq-F + Tub2-seq-R, a 1,306-bp PCR fragment was amplified from each sequenced isolate. PCR products were purified, ligated into the pMD18-T Vector (TaKaRa Biotech. Co., Dalian, China), and then sequenced by Invitrogen Biotech. Co. Ltd. (Shanghai, China). PCR amplifications were performed three times independently for each isolate to avoid mismatch sequences during PCR amplification and sequencing. The sequences were submitted to GenBank under accession numbers GU338401 to GU338408. The DNA sequence of putative extrons of the Tub2 from each isolate was identified based on the extron sequence of Tub2 which was submitted to the $G$. zeae genome database under accession number FGSG 06611. The nucleotide sequence of extrons was translated into amino acid sequences using EditSeq 5.01 (DNASTAR Inc., Madison, WI). The deduced amino acid sequences were aligned using the program ClustalW1.82 (http://www.ebi.ac.uk/clustalw/; European Bioinformatics Institute, Cambridge, UK).

Design of allele-specific PCR primers for the rapid detection of point mutations in Tub2 mediating carbendazim resistance. Three pairs of allele-specific primers were designed for detection of the point mutations at codons 167,198 , or 200. Based on the single point mutation from $\mathrm{T}$ to $\mathrm{A}$ at codon position 167, a nucleotide $\mathrm{T}$ at the $3^{\prime}$ end of the reverse primer $167-\mathrm{RB}$ was designed to match the A at this codon position in the HR isolates. To improve the specificity of traditional allele-specific PCR amplification, we introduced an artificial mismatch base $\mathrm{T}$ at the second nucleotide at the $3^{\prime}$ end of the

Table 2. List of primers used in this study

\begin{tabular}{lll}
\hline Name & \multicolumn{1}{c}{ Sequence $\left(\mathbf{5}^{\prime} \mathbf{- 3}^{\prime}\right.$ ) } & \multicolumn{1}{c}{ Description $^{\mathbf{a}}$} \\
\hline Tub2-seq-F & ACAAACGCACACACACACACA & PCR primers for amplification of the part of $T u b 2$ \\
Tub2-seq-R & AAGATGGCGGAACAAGCGA & $\ldots$ \\
Tub2-F & TTGTAATTGCAAATTTCAACGTG & PCR primers for specifically detection MBC-resistant isolates \\
167-RB & GCGAGGGCATAACGGATT & $\ldots$ \\
198-RB & CGTTATCGATACAGAAGGTCAG & $\ldots$ \\
200-RB & GCCTCGTTATCGATACATT & $\ldots$ \\
Tub1-up-F & CTCGAGTCTGGTACTGCCAACCCACT & PCR primers for amplification of upstream of $T u b 1$ gene with exogenous enzyme \\
Tub1-up-R & GTCGAGTGCCGTTGTAAACACCATTG & $\ldots$ \\
Tub1-down-F & AAGCTTTCATGTCCGGCGTCACTA & PCR primers for amplification of downstream of $T u b 1$ gene with exogenous enzyme \\
Tub1-down-R & GGATCCTCGTACTCCTCTTCTTCCTCG & $\ldots$ \\
Tub1-id-F & ATCTGCTCTTCCATCTCGTCG & PCR primers for identification of $T u b 1$ deletion mutants \\
Tub1-id-R & TCAAGGGAGCGAATCCGA & $\ldots$ \\
Tub1-RT-F & TTTCTGGCAGACCATCTCTG & Reverse-transcription PCR primers for analysis of expression of Tub1 \\
Tub1-RT-R & AGTTGTCGGGTCGGAAAA & $\ldots$ \\
Tub2-RT-F & CGCAACATGAAGACTTTCCA & Real-time PCR primers for analysis of expression of Tub2 \\
Tub2-RT-R & ACGGAAGTTTGGACGTTGTT & $\ldots$ \\
F-actin-F & ATCCACGTCACCACTTTCAA & PCR primers for amplification of the reference gene actin in real-time PCR \\
F-actin-R & TGCTTGGAGATCCACATTTG & $\ldots$ \\
\hline
\end{tabular}

a $\mathrm{PCR}=$ polymerase chain reaction, $T u b 2=\beta 2$-tubulin gene, $\mathrm{MBC}=$ methyl benzimidazole carbamate fungicides, and Tub1 = $\beta 1$-tubulin gene. 
primer (Table 2) (5). A G at the $3^{\prime}$ end of the reverse primer 198-RB was designed to match the $\mathrm{C}$ at codon 198 in the MR isolate, and an artificial mismatch base $\mathrm{A}$ at the second nucleotide at the $3^{\prime}$ end of the primer was introduced. The nucleotide $\mathrm{T}$ at the $3^{\prime}$ end of the reverse primer 200-RB was designed to match the $\mathrm{A}$ at codon 200 in the HR isolate, and an artificial mismatch base $\mathrm{T}$ at the second nucleotide at the $3^{\prime}$ end of this primer was introduced. The forward primer Tub2-F was designed based on the sequences of the fifth intron of the Tub2 from G. zeae. This forward primer was expected to be specific for $G$. zeae because we observed that the fifth intron sequence of Tub2 in G. zeae was different from those in other closely related fungal species such as Fusarium oxysporum and $F$. verticillioides when the sequences were aligned using the program ClustalW1.82.

To determine the specificity of the allele-specific PCR primers, fungal DNA was extracted from each of the carbendazim-resistant isolates: an additional 459 isolates of G. zeae, F. oxysporum, F. verticillioides, and $F$. culmorum isolated from infected wheat heads. The PCR was performed as described above except for the annealing temperature, which was $58^{\circ} \mathrm{C}$.

Construction of $\beta 1$-tubulin gene deletion mutants. A Tubl deletion vector, pCA-tub1-Del, was constructed by inserting two flanking sequences of the Tubl gene into two sides of the hygromycin resistance $(h p h)$ gene in the pBS-HPH1 vector (10) (Fig. 1A). Briefly, a 481-bp upstream flanking sequence fragment of Tubl amplified from genomic DNA of HR isolate GJ33 using the primers Tub1-up-F + Tub1-up-R (Table 2) was inserted into XhoI-Sal I sites of the pBS-HPH1 vector to generate the plasmid pBS-tub1-up. Subsequently, a 619-bp downstream flanking sequence fragment of Tubl amplified from the GJ33 genomic DNA using the primers Tub1-down-F + Tub1-down-R (Table 2) was inserted into HindIII-BamHI sites of the pBS-tub1-up vector to generate the plasmid pBS-tub1-UD. Finally, the 2,603bp fragment containing the Tub1upstream-hph-Tub1-downstream cassette was obtained by digestion of the plasmid pBS-tub1-UD with XhoI and BamHI and ligated into the XhoI-BamHI sites of pCAMBIA 1300 (CAMBIA, Canberra, Australia). The resultant Tubl deletion vector pCA-tub1-Del (Fig. 1A) was transformed into Agrobacterium tumefaciens strain $\mathrm{C} 58 \mathrm{C} 1$. A. tumefaciens-mediated fungal transformation was performed for the HR isolate GJ33 using a previously described protocol (14). Briefly, A. tumefaciens strain $\mathrm{C} 58 \mathrm{C} 1$ containing an appropriate binary vector was grown at $28^{\circ} \mathrm{C}$ for 2 days in minimal medium (MM) supplemented with kanamycin (100 mg liter ${ }^{-1}$ ). The A. tumefaciens cells were diluted to an optical density of $600 \mathrm{~nm}=0.15$ in induc- tion medium (IM) containing $200 \mu \mathrm{M}$ acetosyringone (AS). The cells were grown for an additional $6 \mathrm{~h}$ before mixing with an equal volume of a fresh $F$. graminearum conidial suspension $\left(1 \times 10^{6}\right.$ conidia/ml $)$. A $200-\mu l$ sample of the mixture was sprayed onto a nylon membrane ( 3 by 3 $\mathrm{cm}$ ) (Millipore Co., Bedford, MA) and plated on IM amended with $200 \mu \mathrm{M}$ AS. After incubation at $20^{\circ} \mathrm{C}$ for 2 days in the dark, the membrane was cut into small pieces ( 3 by $0.1 \mathrm{~cm}$ ), and transferred upside down onto PDA plates supplemented with hygromycin $\mathrm{B}\left(100 \mathrm{mg}\right.$ liter $\left.^{-1}\right)$ and cefotaxime $(200 \mu \mathrm{M})$. After 5 to 7 days, individual transformants were transferred onto fresh PDA plates amended with hygromycin B at $100 \mathrm{mg} \mathrm{liter}^{-1}$.

Tubl deletion mutants were identified by primer pair Tub1-id-F+Tub1-id-R and further by southern hybridization analysis using a standard protocol (17) with the probe indicated in Figure 1A.

Analysis of expression of Tub2 gene in Tub1 deletion mutants. Expression of $T u b 2$ was assessed for the Tub1 deletion mutants and the parent isolate. Five mycelial plugs $(5 \mathrm{~mm})$ of each isolate were inoculated in $100 \mathrm{ml}$ of potato dextrose broth medium and incubated at $25^{\circ} \mathrm{C}$ for 4 days. Before mycelia were harvested for RNA extraction, each mutant was treated with carbendazim at $500 \mathrm{mg}$ a.i. liter $^{-1}$ for $6 \mathrm{~h}$. RNA from fungal mycelia was extracted using a TaKaRa RNAiso Reagent (TaKaRa Biotech. Co.) according to the

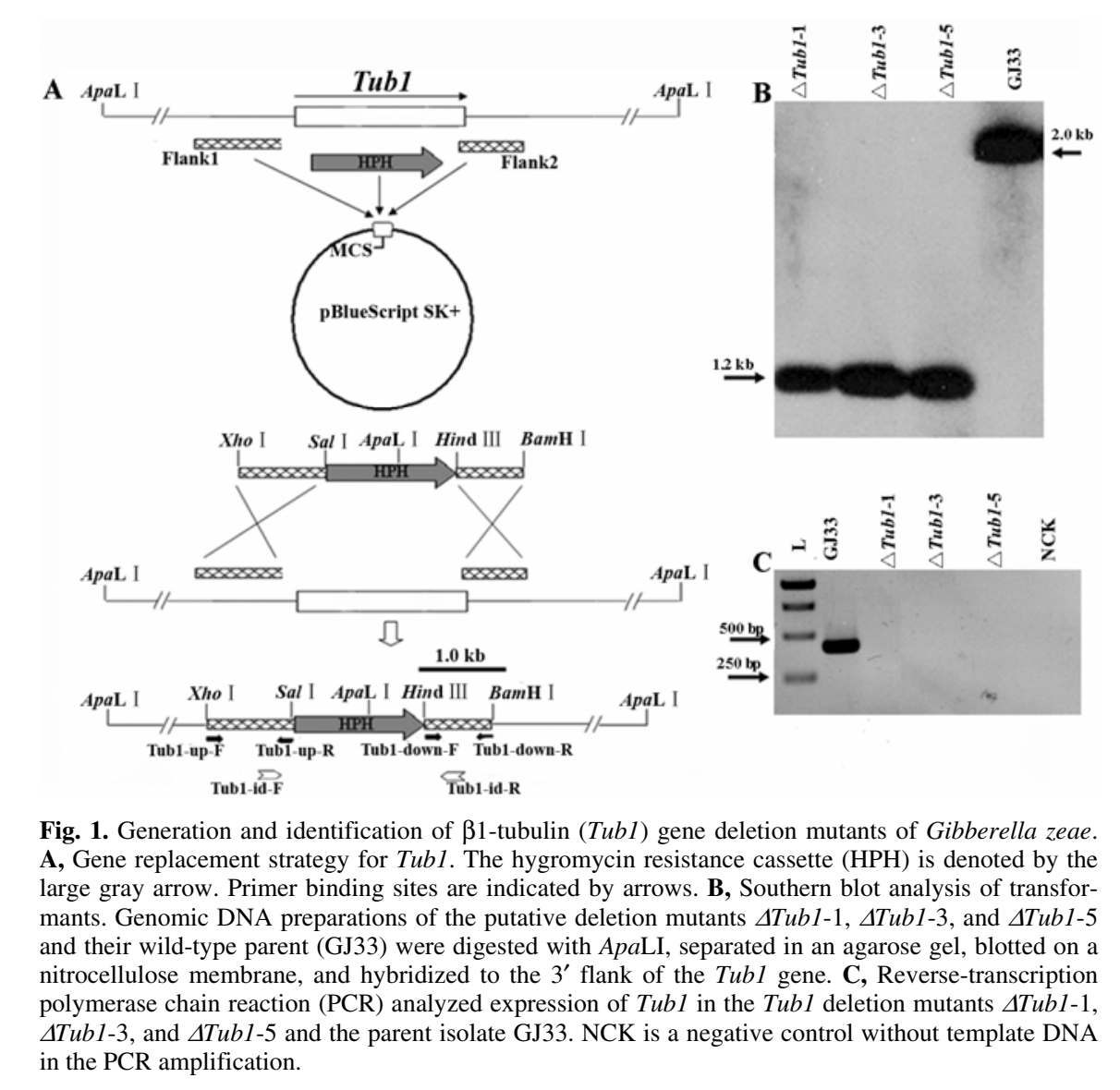

Fig. 1. Generation and identification of $\beta 1$-tubulin (Tub1) gene deletion mutants of Gibberella zeae. A, Gene replacement strategy for Tubl. The hygromycin resistance cassette (HPH) is denoted by the large gray arrow. Primer binding sites are indicated by arrows. B, Southern blot analysis of transformants. Genomic DNA preparations of the putative deletion mutants $\Delta T u b 1-1, \Delta T u b 1-3$, and $\Delta T u b 1-5$ and their wild-type parent (GJ33) were digested with ApaLI, separated in an agarose gel, blotted on a nitrocellulose membrane, and hybridized to the $3^{\prime}$ flank of the Tub1 gene. C, Reverse-transcription polymerase chain reaction (PCR) analyzed expression of Tub1 in the Tub1 deletion mutants $\triangle T u b 1-1$, $\triangle T u b 1-3$, and $\triangle T u b 1-5$ and the parent isolate GJ33. NCK is a negative control without template DNA in the PCR amplification. manufacturer's instructions, and cDNA was synthesized by using a RevertAid $\mathrm{H}$ (Fermentas Canada) with the oligo $(\mathrm{dT})_{18}$ primer. The expression levels of Tub2 were analyzed with real-time PCR assays using the primers Tub2-RT-F + Tub2-RT-R and the SYBR Green I fluorescent dye in a DNA Engine Opticons 4 System (MJ Research, actin-F + F-actin-R (Table 2) for the quantification of expression of actin gene were performed as a reference. The experiment was repeated three times. The expression of $T u b 2$ in the $T u b 1$ deletion mutant relative to that of the parent isomethod (9). The analysis of variance procedure of SAS (version 8.0; SAS Institute, Cary, NC) was used to determine significant differences in expression of Tubl deletion mutants.

\section{RESULTS}

Sensitivity of $G$. zeae to carbendazim. Among 1,529 isolates, 31 were able to (1- to 3-mm colony diameter) $\mathrm{mg}$ a.i. liter $^{-1}$. The $\mathrm{EC}_{50}$ values for these 31 isolates ranged from 10.35 to $30.26 \mathrm{mg}$ a.i. liter $^{-1}$, with a mean of $21.04 \mathrm{mg}$ a.i. liter ${ }^{-1}$ (Table 3). These isolates were designated amplifica, MA). For each sample, PCR as HR isolates. Ten MR isolates were able 
to grow slowly (3- to 5-mm colony diameter) on PDA amended with carbendazim at $100 \mathrm{mg}$ a.i. liter $^{-1}$ but not at $500 \mathrm{mg}$ a.i. liter $^{-1}$. The $\mathrm{EC}_{50}$ values for these $10 \mathrm{MR}$ isolates ranged from 4.50 to $7.28 \mathrm{mg}$ a.i. liter $^{-1}$, with a mean of $5.80 \mathrm{mg}$ a.i. liter $^{-1}$ (Table 3). The remaining 1,488 isolates of G. zeae were unable to grow with carbendazim at $2 \mathrm{mg}$ a.i. liter $^{-1}$ and they were considered sensitive $(S)$ to the fungicide. $\mathrm{EC}_{50}$ values for 10 randomly selected $\mathrm{S}$ isolates ranged from 0.56 to $0.64 \mathrm{mg}$ a.i. liter $^{-1}$, with a mean of $0.59 \mathrm{mg}$ a.i. liter $^{-1}$.

Point mutations in Tub2 from HR and MR isolates. Analysis of DNA sequences of Tub2 showed that the codon TTT at position 167 was replaced by the codon TAT (F167Y) in three HR isolates (GJ33, HA29, and HN40). The codon TTC at the position 200 was replaced by TAC (F200Y) in three other HR isolates (HA36, HA70, and HY11). In the two MR isolates (HA34 and XS19), a point mutation (E198Q) was detected. The codon GAG for glutamic acid at the position 198 was changed to CAG for glutamine in the MR isolate (Fig. 2). Apart from these mutations at positions 167, 198, and 200, all nine isolates analyzed had identical deduced amino acid sequences of Tub2.

Table 3. Point mutation in the Tub 2 from carbendazim-resistant isolates of Gibberella zeae and their $\mathrm{EC}_{50}$ values to carbendazim ${ }^{\mathrm{a}}$

\begin{tabular}{|c|c|c|c|c|}
\hline Isolates & $\begin{array}{c}\text { Origin } \\
\text { (city, province) }\end{array}$ & $\begin{array}{c}\mathbf{E C}_{50} \\
\text { (mg a.i. liter-1) }\end{array}$ & $\begin{array}{c}\text { Resistance } \\
\text { level }^{\mathrm{b}}\end{array}$ & $\begin{array}{l}\text { Mutation in } \\
\text { Tub2 }\end{array}$ \\
\hline $\mathrm{HN} 27$ & Haining, Zhejiang & $0.62 \pm 0.06$ & $\mathrm{~S}$ & No mutation \\
\hline HB61 & Zhaoxian, Hebei & $0.63 \pm 0.15$ & $\mathrm{~S}$ & No mutation \\
\hline AH41 & Ningguo, Anhui & $0.63 \pm 0.07$ & $\mathrm{~S}$ & No mutation \\
\hline AH64 & Liu'an, Anhui & $0.64 \pm 0.03$ & $\mathrm{~S}$ & No mutation \\
\hline GJ37 & Hai’an, Jiangsu & $0.62 \pm 0.16$ & $\mathrm{~S}$ & No mutation \\
\hline HN18 & Haining, Zhejiang & $0.61 \pm 0.07$ & $\mathrm{~S}$ & No mutation \\
\hline HUBEI121 & Jingzhou, Hubei & $0.60 \pm 0.09$ & $\mathrm{~S}$ & No mutation \\
\hline HZ0921 & Hangzhou, Zhejiang & $0.56 \pm 0.12$ & $\mathrm{~S}$ & No mutation \\
\hline LJ2092 & Fuyang, Anhui & $0.57 \pm 0.01$ & $\mathrm{~S}$ & No mutation \\
\hline TA86 & Tai'an, Shandong & $0.59 \pm 0.09$ & $\mathrm{~S}$ & No mutation \\
\hline GHD19 & Hai' an, Jiangsu & $6.22 \pm 1.41$ & MR & E198Q \\
\hline GHD27 & Hai'an, Jiangsu & $6.30 \pm 1.06$ & MR & E198Q \\
\hline HA12 & Hai'an, Jiangsu & $5.32 \pm 1.41$ & MR & E198Q \\
\hline HA120 & Hai'an, Jiangsu & $6.14 \pm 0.13$ & MR & E198Q \\
\hline HA123 & Hai'an, Jiangsu & $6.26 \pm 0.13$ & MR & E198Q \\
\hline HA134 & Hai’an, Jiangsu & $4.82 \pm 0.01$ & MR & E198Q \\
\hline HA163 & Hai'an, Jiangsu & $4.50 \pm 0.19$ & MR & E198Q \\
\hline HA168 & Hai'an, Jiangsu & $7.18 \pm 0.14$ & MR & E198Q \\
\hline HA34 & Hai'an, Jiangsu & $5.79 \pm 0.15$ & MR & E198Q \\
\hline XS9 & Xiaoshan, Zhejiang & $7.28 \pm 0.10$ & MR & E198Q \\
\hline HA70 & Hai'an, Jiangsu & $27.86 \pm 2.11$ & HR & F200Y \\
\hline HA36 & Hai'an, Jiangsu & $30.26 \pm 1.06$ & $\mathrm{HR}$ & F200Y \\
\hline HY11 & Haiyan, Zhejiang & $20.98 \pm 1.29$ & HR & F200Y \\
\hline HY215 & Haiyan, Zhejiang & $18.64 \pm 4.24$ & $\mathrm{HR}$ & F167Y \\
\hline GHD29 & Hai'an, Jiangsu & $20.53 \pm 4.43$ & HR & F167Y \\
\hline GHG34 & Hai'an, Jiangsu & $16.23 \pm 1.41$ & $\mathrm{HR}$ & F167Y \\
\hline GJ23 & Jingjiang, Jiangsu & $15.84 \pm 0.39$ & $\mathrm{HR}$ & F167Y \\
\hline GJ33 & Jingjiang, Jiangsu & $20.55 \pm 1.03$ & HR & F167Y \\
\hline HA29 & Hai'an, Jiangsu & $17.37 \pm 1.06$ & HR & F167Y \\
\hline HA131 & Hai'an, Jiangsu & $28.23 \pm 4.40$ & HR & F167Y \\
\hline HA166 & Hai'an, Jiangsu & $28.03 \pm 1.73$ & $\mathrm{HR}$ & F167Y \\
\hline HY213 & Haiyan, Zhejiang & $23.04 \pm 2.14$ & HR & F167Y \\
\hline HY23 & Haiyan, Zhejiang & $10.35 \pm 0.39$ & HR & F167Y \\
\hline HY310 & Haiyan, Zhejiang & $19.67 \pm 0.71$ & $\mathrm{HR}$ & F167Y \\
\hline HY412 & Haiyan, Zhejiang & $17.91 \pm 3.06$ & HR & F167Y \\
\hline HY414 & Haiyan, Zhejiang & $26.49 \pm 4.24$ & HR & F167Y \\
\hline HY524 & Haiyan, Zhejiang & $19.48 \pm 3.53$ & HR & F167Y \\
\hline HY535 & Haiyan, Zhejiang & $19.12 \pm 0.37$ & $\mathrm{HR}$ & F167Y \\
\hline YS0935 & Fuyang, Anhui & $19.94 \pm 0.36$ & HR & F167Y \\
\hline $\mathrm{ZJ} 127$ & Haining, Zhejiang & $19.25 \pm 1.43$ & $\mathrm{HR}$ & F167Y \\
\hline ZJ218 & Haiyan, Zhejiang & $27.40 \pm 0.87$ & $\mathrm{HR}$ & F167Y \\
\hline ZJ312 & Haiyan, Zhejiang & $17.43 \pm 0.45$ & HR & F167Y \\
\hline ZJ322 & Haiyan, Zhejiang & $17.93 \pm 0.02$ & HR & F167Y \\
\hline ZJ52 & Jiaxing, Zhejiang & $18.45 \pm 0.47$ & HR & F167Y \\
\hline ZJ57 & Jiaxing, Zheiiang & $21.86 \pm 4.14$ & HR & F167Y \\
\hline ZJ59 & Jiaxing, Zhejiang & $19.49 \pm 5.02$ & $\mathrm{HR}$ & F167Y \\
\hline ZJHN10 & Haining, Zhejiang & $23.22 \pm 5.05$ & $\mathrm{HR}$ & F167Y \\
\hline ZJHN4 & Haining, Zhejiang & $16.62 \pm 1.14$ & $\mathrm{HR}$ & F167Y \\
\hline HN40 & Haining, Zhejiang & $20.91 \pm 1.70$ & HR & F167Y \\
\hline ZJHN49 & Haining, Zhejiang & $26.03 \pm 2.79$ & $\mathrm{HR}$ & F167Y \\
\hline ZJHN5 & Haining, Zhejiang & $23.26 \pm 3.03$ & HR & F167Y \\
\hline
\end{tabular}

${ }^{\mathrm{a}} \mathrm{EC}_{50}=$ fungicide concentration that results in $50 \%$ inhibition of mycelial growth.

${ }^{\mathrm{b}} \mathrm{S}$, MR, and HR indicate that the isolates are sensitive, moderately resistant, and highly resistant to carbendazim, respectively.

Allele-specific PCR primers for the detection of point mutations in $T u b 2$. Using the primer pairs Tub2-F + 167-RB and Tub2-F $+200-\mathrm{RB}$, the expected 398or 498-bp fragments were amplified only from HR isolates containing changes at codon positions 167 and 200, respectively, but not from any MR or $\mathrm{S}$ isolates of $G$. zeae or any isolates of $F$. oxysporum, $F$. verticillioides, or $F$. culmorum. Using the primer pair Tub2-F + 167-RB, a 398-bp fragment was obtained from 28 of $31 \mathrm{HR}$ isolates, and a 498-bp fragment was amplified by the primer pair Tub2-F + 200-RB from the remaining $3 \mathrm{HR}$ isolates. The results indicated that the HR isolates contained a point mutation at either codon 167 or 200. The primer pair Tub2-F + 198-RB amplified a 494-bp fragment only from the MR isolates but not from any $\mathrm{HR}$ or $\mathrm{S}$ isolates of $G$. zeae or any isolates of $F$. oxysporum, $F$. verticillioides, or $F$. culmorum. Using this pair of primers, the 494-bp fragment was amplified from each of $10 \mathrm{MR}$ isolates (Table 3), suggesting that these isolates had a point mutation at the amino acid position 198 of the Tub2.

Sensitivity of Tub1 deletion mutants to carbendazim. Southern hybridization patterns confirmed that the Tubl deletion mutants ( $\Delta T u b 1-1, \Delta T u b 1-3$, and $\Delta T u b 1-5)$ were the result of a single homologous recombination event at the Tubl locus (Fig. 1B). Reverse-transcription PCR with primer pair Tub1-RT-F + Tub1-RT-R showed that the Tubl gene was not transcribed in the Tub 1 deletion mutants (Fig. 1C).

The Tubl deletion mutants were more resistant to carbendazim than their parent isolate GJ33, although they were morphologically indistinguishable from the parent isolate on PDA plates (Fig. 3). The $\mathrm{EC}_{50}$ for the parent isolate GJ33 was $20.6 \mathrm{mg}$ a.i. liter ${ }^{-1}$, and those for the Tubl deletion mutants $\Delta T u b 1-1, \Delta T u b 1-3$, and $\Delta T u b 1-5$ were 108.6, 100.7, and $113.6 \mathrm{mg}$ a.i. liter $^{-1}$, respectively. Results were observed from $T u b 1$ deletion mutants similar to those obtained from the parent isolate XS9 having a point mutation at codon 198 (data not shown).

Expression of Tub2 in the Tub1 deletion mutants. Real-time PCR assays showed that the expression levels of $T u b 2$ in $\Delta T u b 1-1, \Delta T u b 1-3$, and $\Delta T u b 1-5$ were $76.0,45.5$, and $11.8 \%$, respectively, higher than that in the parent isolate GJ33 (Fig. 4).

\section{DISCUSSION}

In this study, we tested 1,529 isolates and found that 41 were resistant to carbendazim. Among the 41 isolates, 40 were obtained from Zhejiang and Jiangsu Provinces (Table 1), and the frequencies of resistant isolates in the sampled populations ranged from 2 to $40 \%$. Because the carbendazim resistance in $G$. zeae has been fairly common in these regions, growers 
have started applying tebuconazole, not carbendazim, for the control of FHB. In Anhui Province, no resistant isolates were found in 2008 and one was detected in 2009 (Table 1); however, five resistant isolates have been obtained in 2010 ( $\mathrm{J}$. Jiang and Z. Ma, unpublished data), which indicated that the resistance has started developing. Because the resistant populations could develop rapidly under the se- lection pressure of carbendazim, we have suggested that growers apply a mixture of carbendazim and triadimefon for the control of FHB in this region and other provinces, including Hebei, Henan, and Shandong.

Resistance to benzimidazole fungicides has been detected in many fungal species. In most cases, resistance was correlated with single-point mutations in the $\beta$ -

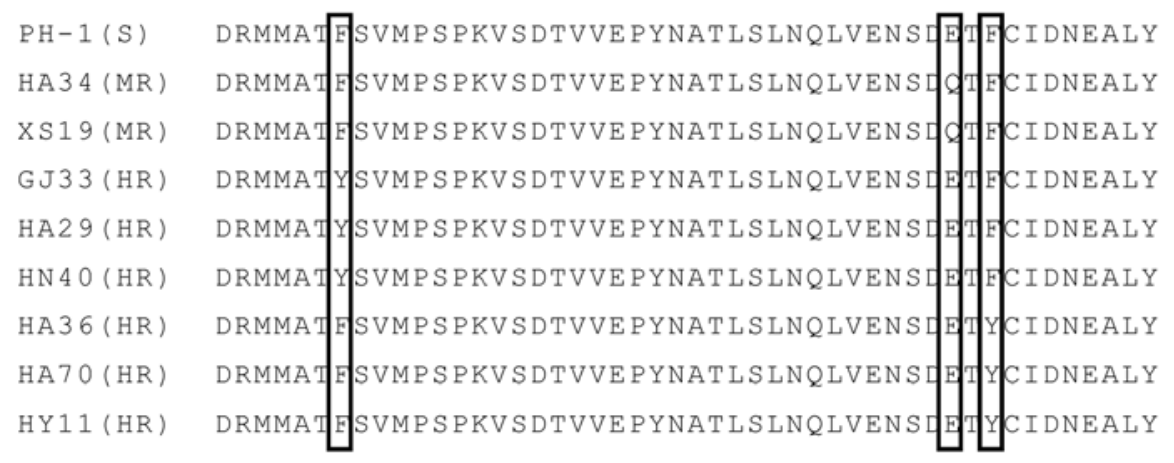

Fig. 2. Alignment of the deduced amino acid sequence (positions 161 to 208) of the $\beta 2$-tubulin (Tub2) gene from carbendazim-sensitive (S), moderately resistant (MR), and highly resistant (HR) isolates of Gibberella zeae. Vertical box indicates the changes in amino acid positions 167, 198, and 200 that were correlated with resistance to carbendazim.

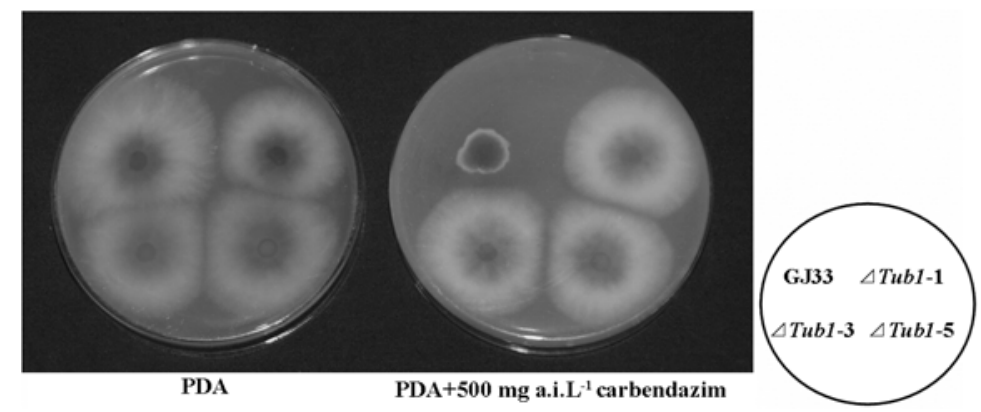

Fig. 3. Comparisons in sensitivity to carbendazim between the $\beta 1$-tubulin $(T u b 1)$ gene deletion mutants ( $\Delta T u b 1-1, \Delta T u b 1-3$, and $\Delta T u b 1-5)$ and parent isolate HR GJ33 of Gibberella zeae.

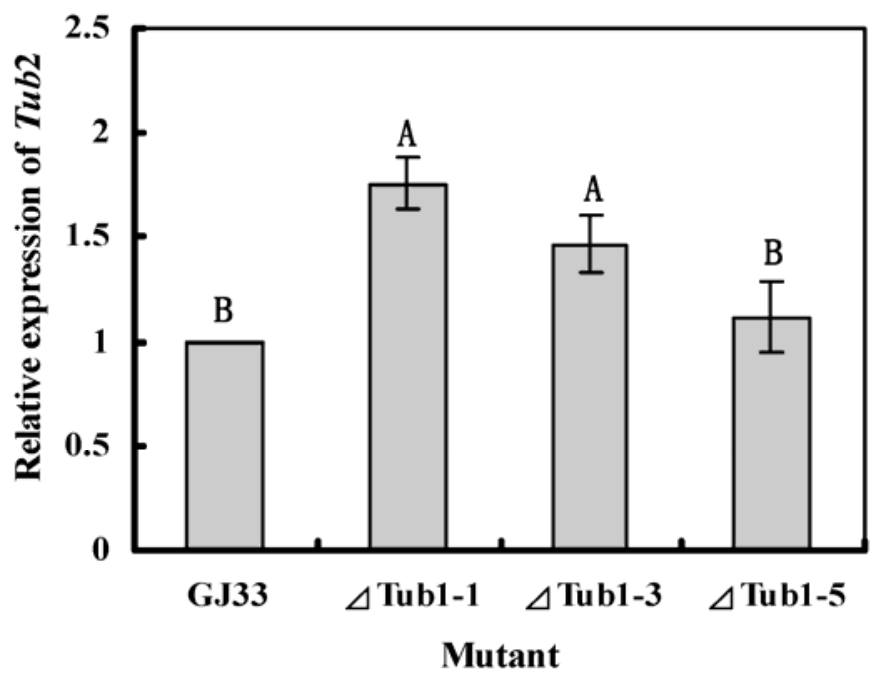

Fig. 4. Comparisons in expression level of $\beta 2$-tubulin (Tub2) between the $\beta 1$-tubulin (Tub1) gene deletion mutants $(\Delta T u b 1-1, \Delta T u b 1-3$, and $\Delta T u b 1-5)$ and their parent isolate GJ33 of Gibberella zeae. Relative expression of Tub2 is the relative amount of Tub2 mRNA compared with the mRNA level from the parent isolate GJ33. Values on the bars followed by the same letter are not significantly different at $P=0.05$. tubulin gene that encodes the target at which the fungicide binds (12). In this study, we detected three single-point mutations (F167Y, E198Q, and F200Y) in the Tub2 from 41 carbendazim-resistant isolates of G. zeae. The isolates with $\mathrm{F} 167 \mathrm{Y}$ and E198Q mutations accounted for $92.6 \%$ of the resistant population, which indicated that these genotypes were predominant in the collected samples.

In this study, we also observed that single-point mutations at codon 167 or 200 of Tub2 caused a high level of resistance, and the point mutation at codon 198 was associated with a moderate level of resistance to carbendazim in $G$. zeae. In a previous study, double-point mutants at codons 73 and 198 were detected in HR isolate JT04. These results indicate that mutations at different codons and different substitutions at the same codon in the $\beta$-tubulin gene may result in different levels of resistance to carbendazim in G. zeae, as has been shown with other fungal species (12).

Many fungi have only a single $\beta$-tubulin gene in their genomes. The presence of two homologous $\beta$-tubulin genes was reported in Aspergillus nidulans, Trichoderma spp., and Colletotrichum spp. $(2,13,15)$. In these species, mutation in one of the $\beta$-tubulin genes was found to be associated with MBC resistance $(6,13,15)$ but the functions of the other $\beta$-tubulin gene have not been documented. This present study is the first to report the contributions of both $\beta$-tubulin genes in sensitivity to MBC fungicide. Targeted disruption experiments showed that the Tubl deletion mutants were morphologically indistinguishable from the parent isolate on PDA (Fig. 3). Interestingly, tests for sensitivity to carbendazim showed that the three Tubl deletion mutants were more resistant to carbendazim. This phenomenon could be partially explained by a higher expression level of Tub2 in the Tub1 deletion mutants. Because there is a point mutant in Tub2 associated with MBC resistance, a higher level of expression of $T u b 2$ in the Tub1 deletion mutants may lead to a higher level of MBC resistance. These results indicate that Tubl may interact with $T u b 2$ in G. zeae but the exact molecular mechanisms for the interactions remain unclear.

\section{ACKNOWLEDGMENTS}

The research was supported by the earmarked fund for Modern Agro-Industry Technology Research System, the Special Program for Agricultural Research (nyhyzx07-048), the 863 project (2007AA10Z422), and the CNSF grant 30900930 .

\section{LITERATURE CITED}

1. Albertini, C., Gredt, M., and Leroux, P. 1999. Mutations of the $\beta$-tubulin gene associated with different phenotypes of benzimidazole resistance in the cereal eyespot fungi Tapesia yallundae and Tapesia acutormis. Pestic. Biochem. Physiol. 64:17-23.

2. Berl, R. O. 2004. Tubulins in Aspergillus nidulans. Fungal Genet. Biol. 41:420-427. 
3. Chen, C., Wang, J., Luo, Q., Yuan, S., and Zhou, M. 2007. Characterization and fitness of carbendazim-resistant strains of Fusarium graminearum (wheat scab). Pest Manage. Sci. 63:1201-1207.

4. Chen, C., Yu, J., Bi, C., Zhang, Y., Xu, J., Wang, J., and Zhou, M. 2009. Mutations in a beta-tubulin confer resistance of Gibberella zeae to benzimidazole fungicides. Phytopathology 99:1403-1411.

5. Drenkard, E., Richter, B. G., Rozen, S., Stutius, L. M., Angell, N. A., Mindrinos, M., Cho, R. J., Oefner, P. J., Davis, R. W., and Ausubel, F. M. 2004. A simple procedure for the analysis of single nucleotide polymorphisms facilitates map-based cloning in Arabidopsis. Plant Physiol. 124:1483-1492.

6. Goldman, G. H., Temmerman, W., Jacobs, D., Contreras, R., Van, Montagu, M., and Herrera, A. 1994. A nucleotide substitution in one of the fl-tubulin genes of Trichoderma viride confers resistance to the antimitotic drug methyl benzimidazole-2-yl-carbamate. Mol. Gen. Genet. 240:73-80

7. Koenraadt, H., Somerville, S. C., and Jones, A. L. 1992. Characterization of mutations in the beta-tubulin gene of benomyl-resistant field strains of Venturia inaequalis and other plant pathogenic fungi. Phytopathology 82:13481354

8. Leroux, P., Fritz, R., Debieu, D., Albertini, C., Lanen, C., Bach, J., Gredt, M., and Chapeland, F. 2002. Mechanisms of resistance to fungicides in field strains of Botrytis cinerea. Pest Manage. Sci. 58:876-888.

9. Livak, K. J., and Schmittgen, T. D. 2001. Analysis of relative gene expression data using real-time quantitative $\mathrm{PCR}$ and the $2^{-\Delta \Delta \mathrm{Ct}}$ method. Methods 25:402-408.

10. Liu, X., Lu, J. P., Zhang, L., Dong, B., Min, H., and Lin, F. C. 2007. Involvement of a Magnaporthe grisea serine/threonine kinase gene, $\operatorname{Mg} A T G 1$, in appressorium turgor and pathogenesis. Eukaryot. Cell 6:977-1005.

11. Luo, Q. Q., Xu, J. Q., Hou, Y. P., Chen, C. J., Wang, J. X., and Zhou, M. G. 2009. PIRAPCR for detection of Fusarium graminearum genotypes with moderate resistance to carbendazim. Plant Pathol. 58:882-887.

12. Ma, Z., and Michailides, T. J. 2005 Advances in understanding molecular mechanisms of fungicide resistance and molecular detection of resistant genotypes in phytopathogenic fungi. Crop Prot. 24:853-863.

13. Maymon, M., Zveibil, A., Pivonia, S., Minz, D., and Freeman, S. 2006. Identification and characterization of benomyl-resistant and -sensitive populations of Colletotrichum gloeosporioides from statice (Limonium spp.) Phytopathology 96:542-548.

14. Mullins, E. D., Chen, X., Romaine, P., Raina, R., Geiser, D. M., and Kang, S. 2001. Agro bacterium-mediated transformation of Fusa rium oxysporum: an efficient tool for insertional mutagenesis and gene transfer. Phytopathology 91:173-180.

15. Nakaune, R., and Nakano, M. 2007. Benomyl resistance of Colletotrichum acutatum is caused by enhanced expression of b-tubulin 1 gene regulated by putative leucine zipper protein CaBEN1. Fungal Genet. Biol. 44:13241335

16. Saitoh, K., Togashi, K., Arie, T., and Teraoka, T. 2006. A simple method for a minipreparation of fungal DNA. J. Gen. Plant Pathol. 72:348-350.

17. Sambrook J., and Russell, D. 2001. Molecular Cloning: A Laboratory Manual, 3rd ed. Cold Spring Harbor Laboratory Press, Cold Spring Harbor, NY.

18. Zhu, Y. Q., Yang, R. M., Diao, C. Y., and Zhu, X. M. 2003. Analysis of causes of disaster of Gibberella zeae in Jiangsu Province in 2003. Plant Prot. Technol. Ext. 23:9-11. 